\title{
A Polar Map Based Approach Using Retinal Fundus Images for Glaucoma Detection
}

\author{
Akshaya Ramaswamy ${ }^{1}$, Keerthi Ram², Niranjan Joshi², Mohanasankar \\ Sivaprakasam ${ }^{1,2}$ \\ ${ }^{1}$ Indian Institute of Technology (IIT) Madras, India \\ ${ }^{2}$ Healthcare Technology Innovation Centre (HTIC), India
}

\begin{abstract}
Cup-to-disc ratio is commonly used as an important parameter for glaucoma screening, involving segmentation of the optic cup on fundus images. We propose a novel polar map representation of the optic disc, using a combination of supervised and unsupervised cup segmentation techniques, for detection of glaucoma. Instead of performing hard thresholding on the segmentation output to extract the cup, we consider the cup confidence scores inside the disc to construct a polar map, and extract sector-wise features for learning a glaucoma risk probability (GRP) for the image. We compare the performance of GRP vis-à-vis the cup-to-disc ratio (CDR). On an evaluation dataset of 100 images from the publicly available RIM-ONE database, our method achieves $82 \%$ sensitivity at $84 \%$ specificity, and $96 \%$ sensitivity at $60 \%$ specificity (AUC of 0.8964 ). Experiments indicate that the polar map based method can provide a more discriminatory glaucoma risk probability score compared to CDR.
\end{abstract}

\section{Introduction}

Glaucoma is a disease that causes optic nerve damage, and can lead to permanent vision loss. Early detection of its presence is the key to prevent complete vision loss. Glaucoma is characterized by structural damage, observed by optic disc imaging, and functional damage observed by visual field examination. Damage in the optic nerve head can mainly be seen in the form of disc cupping, disc haemorrhage, vessel changes inside the optic disc, and peripapillary atrophy around the optic disc. Prior work using monocular retinal fundus images for glaucoma screening can be divided into two main approachessegmentation based approach to extract the disc and cup, and calculate cup-todisc ratio for glaucoma screening; and feature-based approach to extract imagelevel features for glaucoma screening. Following the former approach, many prior methods have focused on achieving accurate cup segmentation to compute the cup-to-disc ratio [1][2][3], but only few [4][5] have explored the next step of using this information for screening. [4] implements a superpixel-based segmentation followed by ellipse fitting, while in [5], thresholding and morphological operations are performed, to extract the optic disc and optic cup boundaries. Various parameters are calculated from these structures and used for image level classification. Methods following the latter approach extract global features like wavelet-based features [6], fractal features [7], and Gabor features [8] for classification of normal and glaucomatous images. Recent works 
have also tried reconstruction based learning techniques [9][10] to compute the optic disc and optic cup parameters for glaucoma detection. Among the works utilizing information from optical coherence tomography (OCT), [11] implements a super-pixel based technique to detect glaucoma using spectral domain (SD)-OCT images, and [12] takes a multi-modal approach by combining information from fundus and SD-OCT images for optic disc and optic cup segmentation.

Our approach utilizes the spatial information from the cup segmentation outputs from fundus images, rather than computing a single cup-to-disc ratio parameter. We map the output confidence scores into a polar representation of the disc. From the polar map, we extract sector-wise features that capture the spatial distribution of the confidence values within the disc, to classify images as normal or glaucomatous.

\section{$2 \quad$ Methodology}

Our proposed method consists of four main steps (refer fig. 1): 1) supervised segmentation to extract cup confidence maps, 2) unsupervised segmentation to extract a binary cup output, 3) polar map representation using the segmentation outputs to compute sector-wise features, and 4) prediction of glaucoma risk probability (GRP).

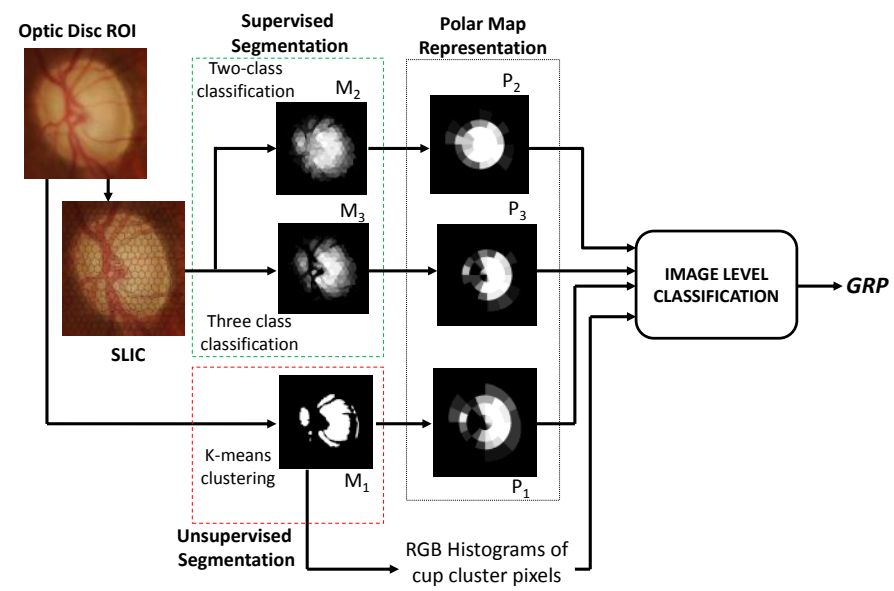

Figure 1: Flow diagram of the polar map based glaucoma screening method

\subsection{Supervised cup segmentation}

Superpixel generation: We implement a supervised segmentation of the optic cup using a superpixel-based method similar to the one proposed in [4]. We use a simple linear iterative clustering (SLIC) technique to generate superpixels on 
the image. This adaptive clustering technique makes use of the $\mathrm{L}, \mathrm{a}^{*}$ and $\mathrm{b}^{*}$ intensity information and the spatial information (location) of the pixels to form superpixels. We set the number of superpixels in such a way that the optic disc contains approximately 100 superpixels. The optic disc boundary is assumed to be given, and further steps are performed only on the superpixels inside the optic disc.

Feature extraction: For each superpixel in the optic disc, we extract histogram based (1280) features, center-surround difference based (36) features, and a distance based feature [4]. We compute the normalized 256-bin contrast enhanced histograms of the red, green, blue, hue and saturation channels. We then obtain nine spatial scale dyadic Gaussian pyramids of each image, and calculate six center surround difference maps in the red, green and blue channels. From these maps, the mean and variance within every superpixel are extracted. Finally, a normalized distance parameter from the disc center to each superpixel centroid is also computed.

Supervised classification: The extracted superpixel features are used to train a classifier $\mathrm{C}_{2}$ to classify the disc into cup and rim superpixels, as implemented in [4]. Using the blood vessel superpixels inside the disc for training can affect the accuracy of the cup-rim classification. So in addition to $C_{2}$, we train another classifier $\mathrm{C}_{3}$ to classify the disc superpixels into cup, rim and blood vessels (three-class). The training data for the blood vessel class is created using a standard blood vessel segmentation algorithm such as [13]. $\mathrm{C}_{2}$ and $\mathrm{C}_{3}$ assign cup probability value to each superpixel inside the disc, to form two cup confidence maps $\mathrm{M}_{2}$ and $\mathrm{M}_{3}$ (refer fig. 2(c) and 2(d)).

\subsection{Unsupervised segmentation}

Cupping in the optic disc is generally identified by the colour contrast and pallor. There are large intensity variations among retinal images, making a single characterization of cupping difficult, compounded by inter-observer variability in cup ground truth demarcation. We therefore utilize the local contrast within the image and perform an unsupervised k-means clustering on the $\mathrm{L}, \mathrm{a}^{*}$ and $\mathrm{b}^{*}$ pixel intensities inside the disc. We set $\mathrm{k}=3$, to extract three clusters corresponding to blood vessels, rim and cup respectively. From the clustering output (refer fig. 2(e)), we extract the binary cup output $M_{1}$ as the brightest cluster. After the segmentation step, for each image we get two confidence maps $\mathrm{M}_{2}$ and $\mathrm{M}_{3}$ from the supervised method, and a binary cup output $\mathrm{M}_{1}$ from the unsupervised method. 

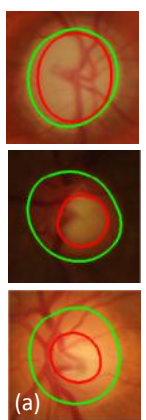
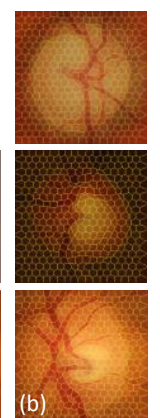
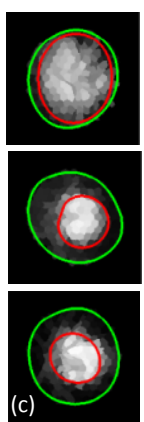
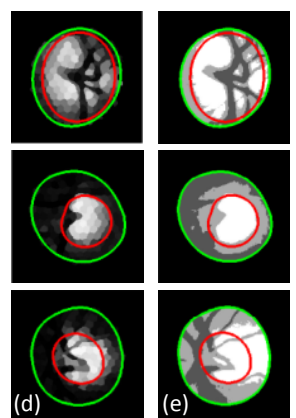

Figure 2: (a) Original image, (b) Superpixel formation, (c) confidence map output of two-class classification $\left(M_{2}\right)$, (d) confidence map output of three-class classification $\left(\mathrm{M}_{3}\right)$, (e) k-means clustering output showing brightest cluster (in white) that gives $M_{1}$; Ground truth indicated (Optic disc: green, optic cup: red)

\subsection{Polar map based feature extraction}

Polar map representation: The risk of having glaucoma varies based on the spatial location and extent of the cup, and is higher in the case of notching in the inferior or superior regions. We use the confidence maps which encode such spatial information, and propose a learning based method to build a model of glaucoma risk. We introduce a polar map representation to extract sector-wise features from $M_{1}, M_{2}$ and $M_{3}$. For each image, we overlay three concentric circles from the disc center at radii $0.2,0.5$ and 0.7 times the maximum disc radius. We consider the optic disc boundary to form the outermost boundary of our polar map. We divide each circle or annulus into 16 sectors, to get a total of 64 sectors (refer fig. 3 (a)).

Polar map features: Using the constructed polar representation, we extract 64 sector-wise features. Using $\mathrm{M}_{2}$ and $\mathrm{M}_{3}$ we average the confidence values inside each sector to generate 64 sector-wise features. From $M_{1}$, we average the binary values inside each sector to get 64 features (refer fig 3 (c-e)). We adopt the left eye frame of reference, and horizontally flip all right eye images before calculating the sector-wise features.
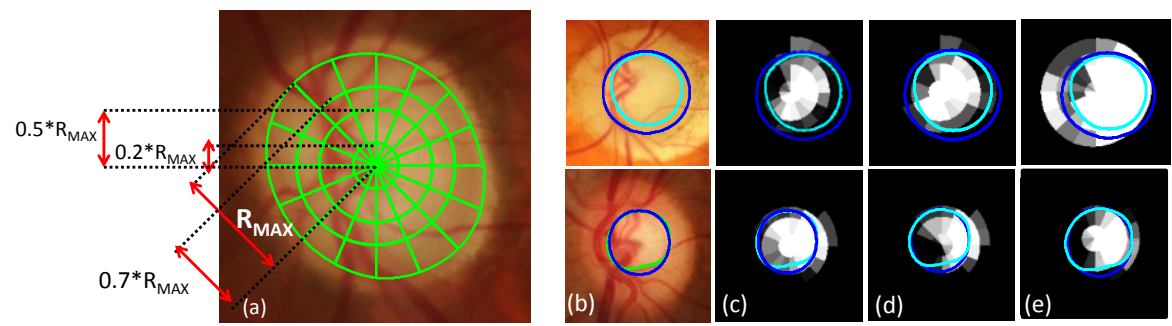

Figure 3: (a)Image indicating the polar map template; (b-e): Inter-observer variability shown using optic cup boundary marked by Expert 1 (blue) and Expert 2 (green) on (b) original image (c) Polar map $P_{2}$ using two-class confidence map output, (d) Polar map $P_{3}$ using three-class confidence map output, (e) Polar map $P_{1}$ using k-means binary map output; 


\section{$2.4 \quad$ Image level glaucoma screening}

From $M_{1}, M_{2}$ and $M_{3}$, we generate three polar maps $P_{1}, P_{2}$ and $P_{3}$, and extract 192 sector-wise features. Along with the polar map-based features, we also consider histogram-based features. We compute 8-bin RGB histograms of the original image pixels corresponding to region in $\mathrm{M}_{1}$. From each image, we extract a total of 216 features, which are given as input to a trained Random Forest classifier for glaucoma detection. The output of the classifier is a glaucoma risk probability (GRP).

\section{Experimental results and discussion}

The proposed polar map based approach is evaluated on a set of 100 cropped optic disc ROIs randomly selected from images in RIM-ONE r1, RIM-ONE r2 and RIM-ONE $r 3$ databases [14]. The 100-image dataset consists of 50 normal images, and 50 glaucomatous images. The image-level ground truth is available for all the images, and the ground truth cup boundaries are available for images taken from RIM-ONE r3. Cup ground truth for the rest of the images was manually marked.

\subsection{Cup Segmentation}

Cup-to-disc ratio, a commonly used parameter for glaucoma screening, can be computed from the confidence maps by thresholding. We use threshold on $\mathrm{M}_{2}$ (confidence map obtained from two-class classification) for cup segmentation. The performance of the cup segmentation using a random forest classifier with 10-fold validation trained with 500 trees and $36(\sqrt{N})$ features selected at random is shown in figure 4 (a). The ROC curve has area-under the curve (AUC) of 0.8928 . On our dataset of 100 images, we perform ellipse fitting on $\mathrm{M}_{2}$, for various thresholds and calculate the Dice Index (refer fig 4 (b)). We find the threshold that gives maximum Dice Index of the fitted ellipse. At this threshold, we calculate the vertical cup-to-disc diameter ratio. Figure 5 shows the ellipse output on two images for different thresholds.
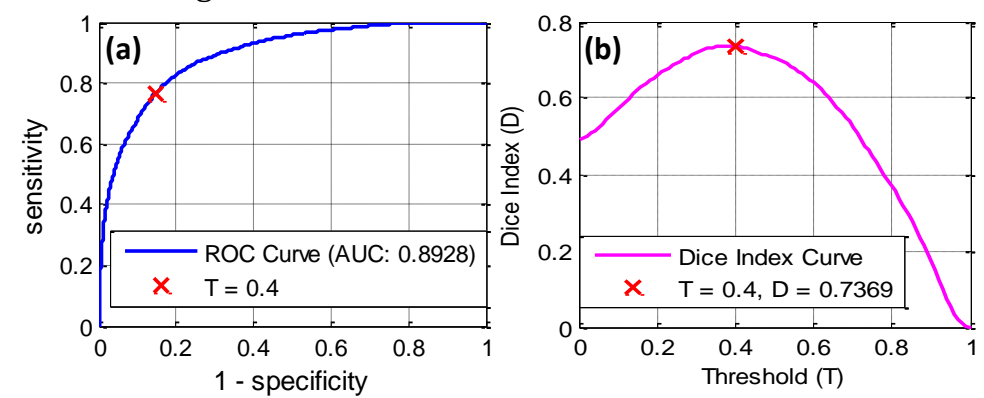

Figure 4: (a) ROC curve (AUC $=0.8928$ ) indicating the performance of $\mathrm{M}_{2}$ (ten-fold validation using a random forest classifier with 500 trees and 36 features selected at random), (b) Curve showing variation of Dice Index with threshold; Maximum Dice index $=0.7369$ at threshold $=0.4$ 

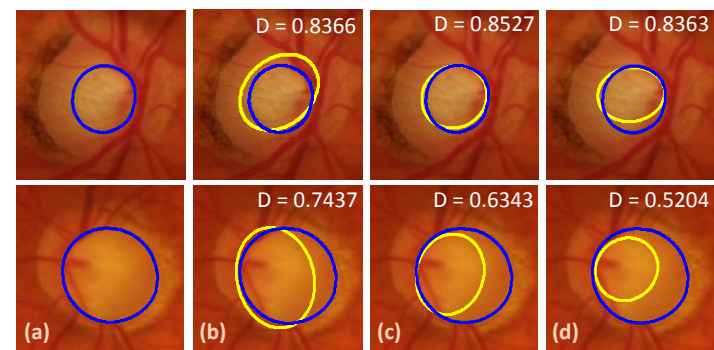

Figure 5: (a) Original image, Cup ground truth (in blue), (b-d) Ellipse fit (in yellow) for and Dice Index (D) for (b) threshold $=0.3$, (c) threshold $=0.4$, (d) threshold $=0.5$

\subsection{Image level classification}

We perform three experiments to evaluate the screening performance of our proposed approach: i) performance evaluation for different groups of imagelevel features derived from the two-class, three-class and k-means output, ii) comparison with CDR-based screening approach, iii) histogram analysis to assess the inter-class separation of the predicted CDRs and that of glaucoma risk probability (GRP).

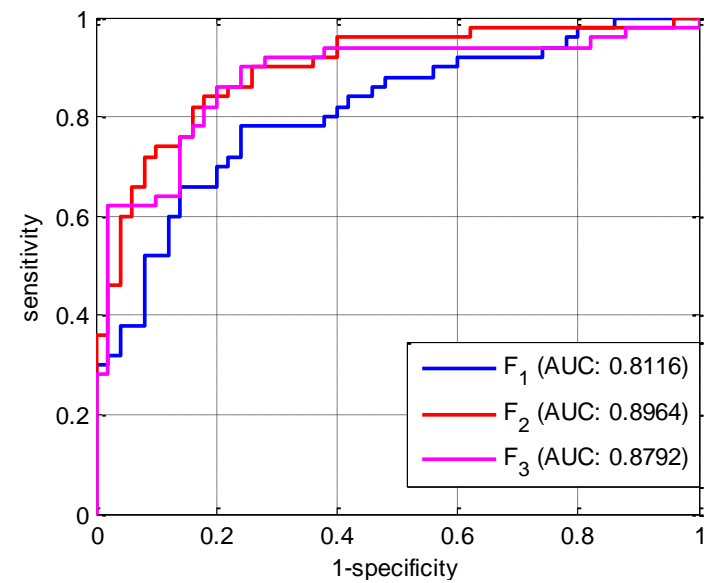

Figure 6: Comparison of ROC curves for leave-one-out validation using different image-level features on 100 images using Random Forest classifier with 1000 trees and 14 variables selected at random; $F_{1}$ (features from $P_{1}$ ), $F_{2}$ (features from $P_{1}, P_{2}$ and $P_{3}$ ), $F_{3}$ (features from $P_{2}$ and $P_{3}$ )

We perform ROC analysis to evaluate the performance of our approach. We implement a leave-one-out validation on the dataset for different groups of image-level features derived from $\mathrm{P}_{1}, \mathrm{P}_{2}$ and $\mathrm{P}_{3}$. We use a random forest classifier trained with 1000 trees and $14(\sqrt{ } N)$ variables selected at random. The three subsets of features evaluated are $\mathrm{F}_{1}$ (features from $\mathrm{P}_{1}$ ), $\mathrm{F}_{2}$ (features from $\mathrm{P}_{1}, \mathrm{P}_{2}$ and $P_{3}$ ) and $F_{3}$ (features from $P_{2}$ and $P_{3}$ ). Combining the image level features 
from all the three segmentation maps (F2) achieves the maximum area under ROC curve (AUC) of 0.8964 (refer fig. 6).

The performance of the proposed polar map based method is compared against the CDR-based method. The CDR-based detection is implemented by thresholding and ellipse fitting on $\mathrm{M}_{2}$ to extract the optic cup, and then computing the cup-to-disc ratio. The calculated CDRs are used to plot the ROC curve for CDR-based detection. The polar map based method using all the features $\left(\mathrm{F}_{2}\right)$ achieves the maximum area under curve (AUC) 0.8964 , while the CDR-based detection achieves an AUC of 0.8416 (refer fig 7). Note that it is possible to combine CDR as one of the features in the polar map based method, to improve the performance further.

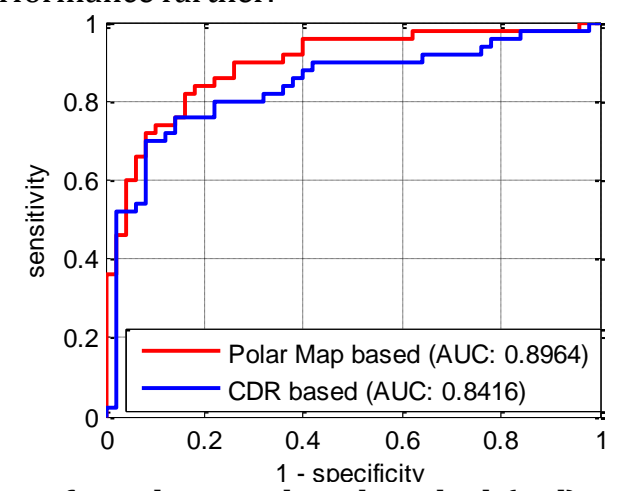

Figure 7: ROC curves for polar map based method (red) and CDR-based method (blue) on the dataset of 100 images

To compare the inter-class separation between normal and glaucomatous images, we plot the histograms of the 100 images for two cases: 1) predicted CDRs, and 2) predicted GRP of the proposed polar map based method (refer fig. 8). We compute the Bhattacharyya measure to assess the similarity between the normal and glaucoma distributions [15]. The value of Bhattacharyya coefficient $\rho_{\mathrm{GRP}} 0.6526$ for the predicted glaucoma risk probability is lower than $\rho_{\mathrm{CDR}} 0.7023$ for the predicted CDR, showing higher inter-class separability for GRP. This indicates that GRP can be a reliable discriminatory parameter to distinguish between normal and glaucomatous images.
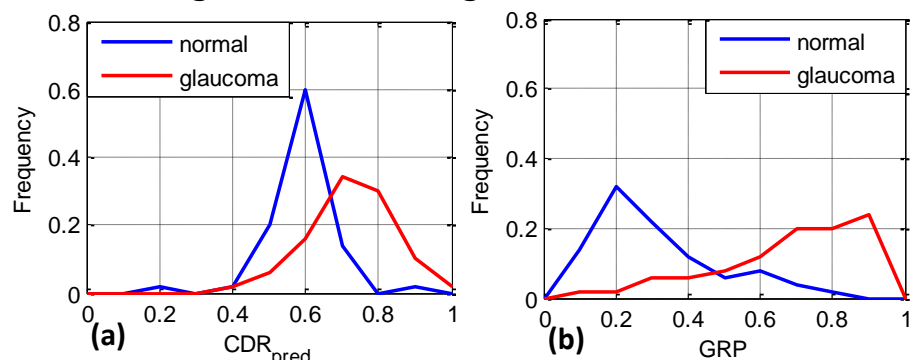

Figure 8: Comparison of the normalized histograms of normal (blue) and glaucomatous (red) images for (a) predicted CDRs ( $\mathrm{CDR}_{\text {pred}}$ ), and (b) glaucoma risk probabilities (GRP) for 100 images 


\section{Conclusion}

We propose a new method that incorporates supervised and unsupervised cup segmentation outputs to construct a polar map. Using this, we extract sectorwise features that are used for glaucoma detection. We compare our method with the CDR based method, and show that the polar map based method gives a better performance with an AUC of 0.8964 on 100 images, achieving $82 \%$ sensitivity at $84 \%$ specificity, and $96 \%$ sensitivity at $60 \%$ specificity. The value of Bhattacharyya coefficient $\rho_{\mathrm{GRP}} 0.6526$ for the predicted GRP is lower than $\rho_{\mathrm{CDR}}$ 0.7023 for the predicted CDR, showing that GRP can be used as a discriminatory parameter to classify images as normal or glaucomatous. In our future work, we aim to capture other signs of glaucoma such as vessel bends and peripapillary atrophy in our polar map representation.

\section{References}

1. Y. Xu et al., "Optic Cup Segmentation for Glaucoma Detection Using Low-Rank Superpixel Representation," in MICCAI 2014.

2. M. S. Haleem, L. Han, J. van Hemert, and B. Li, "Automatic extraction of retinal features from colour retinal images for glaucoma diagnosis: A review," CMIG, 2013.

3. Y. Zheng, D. Stambolian, J. O'Brien, and J. C. Gee, "Optic Disc and Cup Segmentation from Color Fundus Photograph Using Graph Cut with Priors," MICCAI 2013.

4. Jun Cheng et al., "Superpixel Classification Based Optic Disc and Optic Cup Segmentation for Glaucoma Screening", IEEE Trans. Med. Img, 2013.

5. J. Nayak, et al., "Automated Diagnosis of Glaucoma Using Digital Fundus Images," J Med Syst, 2009.

6. Yun, Wong Li et al., "Glaucoma Classification Using Brownian Motion and Discrete Wavelet Transform", Journal of Medical Imaging and Health Informatics, 2014

7. P. Y. Kim, et al, "Novel Fractal Feature-Based Multiclass Glaucoma Detection and Progression Prediction," IEEE Journal of Biomedical and Health Informatics, 2013.

8. U. R. Acharya, et al, "Decision support system for the glaucoma using Gabor transformation," Biomedical Signal Processing and Control, 2015.

9. Y. Xu et al., "Efficient reconstruction-based optic cup localization for glaucoma screening," in MICCAI 2013.

10. J. Cheng et al., "Sparse Dissimilarity-Constrained Coding for Glaucoma Screening," IEEE Trans. Biomed. Engg, 2015.

11. J. Xu et al., "3D optical coherence tomography super pixel with machine classifier analysis for glaucoma detection," in IEEE EMBC, 2011.

12. M. S. Miri et al., "Multimodal Segmentation of Optic Disc and Cup from SD-OCT and Color Fundus Photographs Using a Machine-Learning Graph-Based Approach," IEEE Trans. Med. Img, 2015.

13. T. Walter and J.-C. Klein, "Segmentation of color fundus images of the human retina: Detection of the optic disc and the vascular tree using morphological techniques," in Medical Data Analysis, Springer, 2001.

14. F. Fumero et al., "RIM-ONE: An open retinal image database for optic nerve evaluation," in Intl. Symposium on Computer-Based Medical Systems (CBMS), 2011.

15. P. Elzbieta, Robert P.W. Duin, "The dissimilarity representation for pattern recognition, foundations and applications", No.64, World Scientific, 2005 